\title{
Echo-guided pin-point compression can effectively repair pseudoaneurysms associated with catheter procedure
}

\author{
Toshiaki Yamanaka $^{1 *}$, Yoichi Nakamura ${ }^{2}$, Yusuke Kawai $^{1}$, Sumiko Sato ${ }^{1}$, Kazuaki Mineoi ${ }^{1}$, \\ Tadakatsu Yamada ${ }^{1}$, Hideki Okayama ${ }^{1}$, Yukio Kazatani ${ }^{1}$, Hiroshi Ito $^{3}$

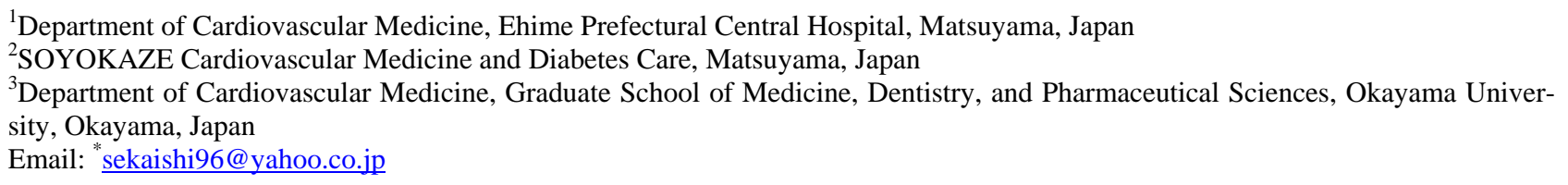

Received 16 May 2012; revised 20 June 2012; accepted 28 June 2012

\begin{abstract}
Background: Catheter intervention is occasionally complicated by a pseudoaneurysm at the puncture site. Although the feasibility of echo-guided repair of a pseudoaneurysm has been reported, this method does not always repair the pseudoaneurysm. We hypothesized that if the communication to the artery could be effectively closed by pin-point compression, the clot that forms in the residual lumen would effectively cover the communication. We studied the safety and efficacy of the echo-guided pinpoint compression procedure for repairing a pseudoaneurysm. Methods: Ten consecutive patients with a pseudoaneurysm were enrolled. We determined the site of communication by echography with a high-frequency linear probe. We performed pin-point compression on the communication point with the right index finger, and we confirmed closure of the communication by color Doppler. During compression, we monitored echo images to confirm clot formation. Results: A pseudoaneurysm was located on the femoral artery in 6 patients and on the brachial artery in 4 patients. The sizes of the pseudoaneurysms ranged from 13 to 40 $\mathrm{mm}$ in diameter. We successfully closed the communication with one-finger compression in all patients. During the compression, we observed clot formation in the residual lumen of the pseudoaneurysm in all patients by echography. The duration of compression ranged from 5 to 40 minutes (mean, 18 minutes). We succeeded in repairing the pseudoaneurysm in all patients using this method. The success of the procedure was also confirmed 24 hours later in all patients. Conclusions: Echo-guided pin-point compression of the communication might be an effective technique for repairing a pseudoaneurysm at the puncture site, and echography is useful for confirming the success
\end{abstract}

\footnotetext{
"Corresponding author.
}

of the procedure.

Keywords: Pseudoaneurysm; Treatment; Ultrasound

\section{INTRODUCTION}

A pseudoaneurysm is a rare complication of a catheter examination or intervention. It is a hematoma that forms as the result of a leaking hole at the puncture site of the artery and is covered by surrounding connective tissues [1]. Because of the communication to the artery, the pseudoaneurysm often grows rapidly, resulting in rupture in some cases [2]. Proper management of a pseudoaneurysm remains an important clinical issue.

Surgery is the gold-standard treatment, but most patients hope for a less invasive treatment. One of the less invasive options is ultrasound-guided compression (Figure 1). This procedure, however, is not always successful despite long compression. In this procedure, we have tried to drain as much of the blood from the pseudoaneurysm as possible by compressing it with an ultrasound probe. However, this can result in insufficient blood within the residual space to make a clot stopper at the communication point. We therefore consider that it is important to have sufficient blood in the residual space to make a clot and to stop the blood flow through the communication in order to enhance clot formation over the communication. For these requirements, we have developed a simple approach for compression guided by echography (Figure 1(c)). Pin-point compression with a finger is used to effectively close the communication. This procedure can effectively close the communication with enhancement of the coagulation cascade of blood in the residual space of the pseudoaneurysm (Figure 1(c)). We monitored the success of this procedure and clot formation with echography to improve its clinical efficacy. In this study, we performed this revised procedure 


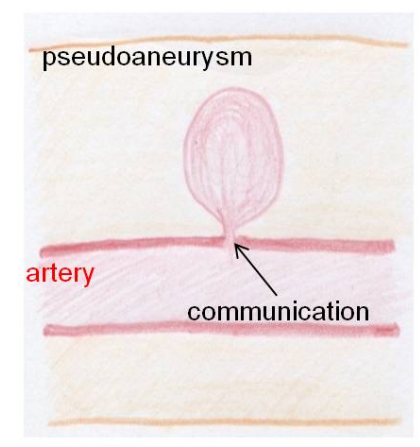

(a)

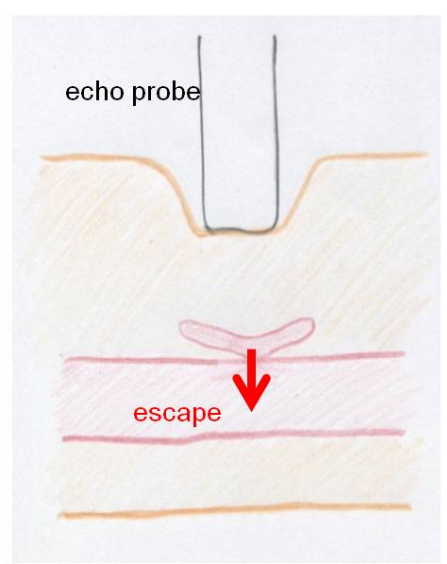

(b)

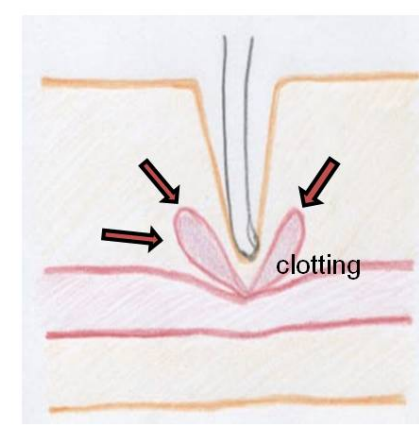

(c)

Figure 1. Pseudoaneurysm at puncture site (a) and methods to treat it (b, c). (a) A pseudoaneurysm forms outside the arterial wall. It is surrounded by connective tissues; (b) Ultrasound-guided compression: A pseudoaneurysm is compressed by an echo-probe. The lumen of the pseudoaneurysm apparently disappears during the compression because the compression procedure drains intra-cavity blood into the artery. There is insufficient blood to make a clot over the communication; (c) Pin-point compression: One-finger compression on the communication point may effectively close the communication. Flow stagnation within the pseudoaneurysm may accelerate the coagulation cascade of blood to effectively make a clot stopper.

in consecutive patients with post-procedural arterial pseudoaneuryms to study its safety and clinical efficacy.

\section{METHODS}

\subsection{Study Population}

In the period from April 2006 to July 2010, 4347 patients underwent catheter examination or intervention in our hospital using the radial artery approach in 2800 patients, brachial artery approach in 356 patients and femoral artery approach in 1191 patients. Ten patients (0.2\%) suffered from pseudoaneurysms and underwent the revised procedure.

Diagnosis of pseudoaneurysm was made with an echo apparatus equipped with a high-frequency linear probe (3 - $13 \mathrm{MHz}$ ) and a color Doppler imaging technique (Figure 2(a)). The entry point, which is the communication to the artery, was identified on the echo images.

\subsection{Procedure for Pin-Point Compression}

Antiplatelet and/or anticoagulant therapies were continued. After identifying the communication to the artery by echography, the right index finger was placed on the pseudoaneurysm just above the communication point (Figure 2(b)), and compression was applied until the flow through the communication stopped, which was confirmed by echography. At that moment, the lumen of pseudoaneurysm had not completely disappeared. The goal of pin-point compression is complete obliteration of flow in the pseudoaneurysm as confirmed by echography. We monitored clot formation within the pseudoaneurysm. After about 5 minutes, the compression was stopped and closure of the communication was confirmed by echography. Flow thorough the communication was reassessed with echography after the procedure. If the flow was still found, pin-point compression was repeated, with the same time increments, until successful pseudoaneurysm thrombosis was achieved as confirmed by echography. We defined clot formation on an echo image as emergence of a heterogeneously echogenic mass together with disappearance of Doppler signal from the sac (Figure 2(c)). Echography was performed 24 hours later to examine the success of the procedure.

\section{RESULTS}

\subsection{Baseline Characteristics}

Table 1 summarizes the clinical data for patients with a pseudoaneurysm undergoing one-finger pin-point compression. Mean age of the patients was 71.4 years (range: 61 - 86 years), and 4 patients were female. Nine patients had hypertension, 6 had dislipidemia, 2 had diabetes, and 2 were undergoing hemodialysis. Nine patients were receiving dual anti-platelet therapy, but none of the patients were receiving anticoagulant therapy. The catheterization procedures included percutaneous coronary intervention in 8 patients, percutaneous trans-arterial angioplasty in one patient, and diagnostic coronary angiography in one patient. Sizes of sheeths used were $5 \mathrm{~F}$ in one patient, $6 \mathrm{~F}$ in 6 patients and 7F in 3 patients. After the catheterization procedures, 7 patients underwent astriction and 3 patients underwent an angioseal procedure. Duration of astriction ranged from 15 to 40 minutes (mean, 27.8 minutes). Six patients had a pseudoaneurysm on the femoral artery and 4 patients had a pseudoaneurysm on the 

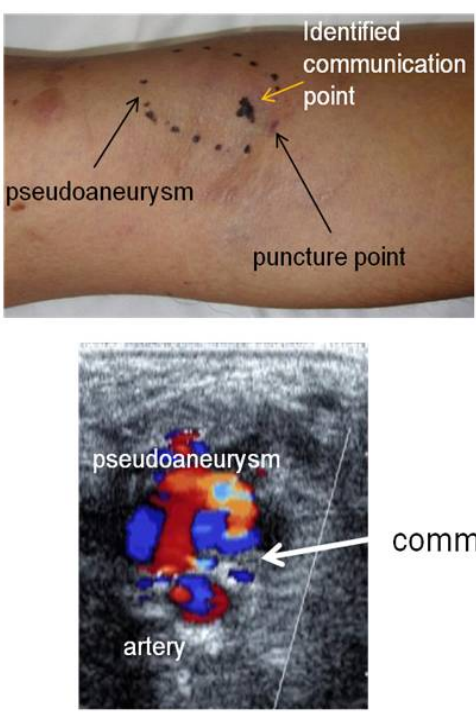

(a)
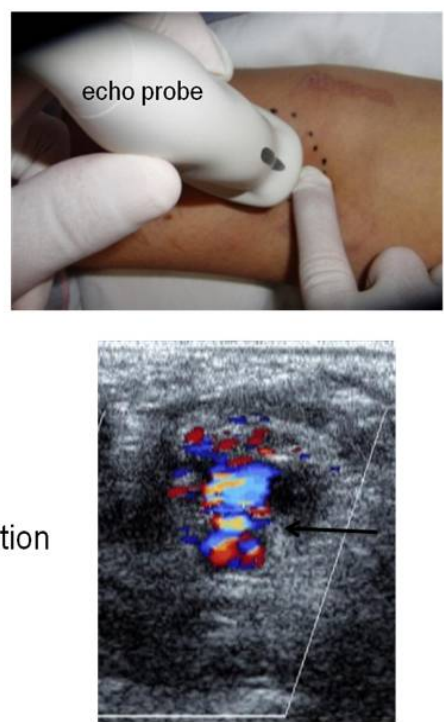

(b)

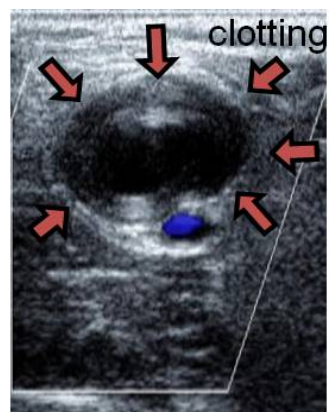

(c)

Figure 2. Echo-guided pin-point compression. (a) This patient had a pseudoaneurym on the brachial artery. We identified the communication to the artery by the color Doppler technique and placed a check mark on the point just above the communication; (b) The right index finger was placed on the check mark and applied pressure until the communication flow stopped. It is important that there is sufficient blood within the residual cavity to make a clot. We also observed the process of clot formation by echography; (c) We confirmed clot formation on an echo image as emergence of an echogenic mass together with disappearance of Doppler signal from the sac.

Table 1. Patients characteristics.

\begin{tabular}{|c|c|c|c|c|c|c|c|c|c|c|c|c|}
\hline No. & Age/Sex & $\begin{array}{l}\text { Height, } \\
\text { cm }\end{array}$ & $\begin{array}{l}\text { Weight, } \\
\text { kg }\end{array}$ & BMI & $\begin{array}{l}\text { Puncture } \\
\text { site }\end{array}$ & $\begin{array}{c}\text { Sheath size, } \\
\text { French }\end{array}$ & $\begin{array}{c}\text { Size of PA, } \\
\mathrm{mm} * \mathrm{~mm}\end{array}$ & $\begin{array}{c}\text { The diameter of } \\
\text { communication, } \\
\text { mm }\end{array}$ & $\begin{array}{l}\text { Onset-Rx } \\
\text { time, days }\end{array}$ & DAT & $\begin{array}{l}\text { Hemostatic } \\
\text { method }\end{array}$ & $\begin{array}{c}\text { Duration of } \\
\text { astriction, } \\
\text { min }\end{array}$ \\
\hline 1 & $74 / F$ & 154 & 55 & 23.3 & brachial & 6 & $10 * 20$ & 2.2 & 1 & + & Astriction & 40 \\
\hline 2 & 76/M & 157 & 54 & 21.9 & femoral & 7 & $25 * 20$ & 3.1 & 3 & + & Angioseal & - \\
\hline 3 & 73/M & 164 & 62 & 23.2 & brachial & 6 & $12 * 20$ & 2.6 & 3 & + & Astriction & 20 \\
\hline 4 & 86/M & 158 & 40 & 16 & femoral & 6 & $15 * 20$ & 2.4 & 1 & + & Astriction & 30 \\
\hline 5 & 77/M & 155 & 63 & 26.4 & femoral & 7 & $8 * 13$ & 1.9 & 3 & + & Angioseal & - \\
\hline 6 & 68/F & 145 & 58 & 27.6 & brachial & 6 & $10 * 18$ & 2.2 & 1 & + & Astriction & 30 \\
\hline 7 & $65 / M$ & 164 & 95 & 35 & femoral & 7 & $30 * 40$ & 4.2 & 1 & + & Astriction & 20 \\
\hline 8 & $72 / F$ & 144 & 47 & 22.9 & femoral & 6 & $20 * 20$ & 2.1 & 1 & + & Astriction & 40 \\
\hline 9 & $62 / \mathrm{M}$ & 163 & 53 & 19.9 & femoral & 6 & $7 * 21$ & 2.7 & 1 & + & Angioseal & - \\
\hline 10 & $61 / \mathrm{F}$ & 154 & 57 & 23.7 & brachial & 5 & $6 * 20$ & 2.3 & 1 & - & Astriction & 15 \\
\hline
\end{tabular}

Abbreviations: $\mathrm{BMI}=$ body mass index; DAT = dual-antiplatelet therapy (aspirin + clopidgrel or ticlopidine or cilostazol); PA = pseudoaneurysm; $\mathrm{Rx}=$ treatment.

brachial artery. Sizes of the pseudoaneyrysm ranged from 13 to $40 \mathrm{~mm}$ in diameter (mean, $19.7 \mathrm{~mm}$ ). Diameters of the communications ranged from 1.9 to $4.2 \mathrm{~mm}$ in diameter (mean, $2.57 \mathrm{~mm}$ ). Time intervals from onset of the pseudoaneurysm to treatment ranged from 1 to 3 days (mean, 1.6 days).

The procedure for pin-point compression is summarized in Table 2. The compression duration ranged from 5 to 40 minutes (mean, 18 minutes). Communication to the artery was successfully identified by the color Doppler technique in all patients. After pin-point compres- sion, the absence of communication flow was confirmed by the color Doppler technique in all patients. Seven patients had severe pain during the compression. The process of clotting in the residual lumen of the pseudoaneurysm was serially assessed by echography. All patients were reexamined by echography 24 hours after the procedure, and it was confirmed that clotting remained. Recurrence of flow through the communication was not observed. The interval between repair of the pseudoaneurysm and hospital discharge ranged from 2 to 12 days (mean, 5.5 days). None of the patients suffered from 
Table 2. Procedure of pin-point compression and results.

\begin{tabular}{|c|c|c|c|c|c|c|c|}
\hline No. & $\begin{array}{l}\text { Identification of } \\
\text { entry with echo }\end{array}$ & $\begin{array}{l}\text { Pain during } \\
\text { compression }\end{array}$ & $\begin{array}{l}\text { Compression } \\
\text { duration, min }\end{array}$ & $\begin{array}{l}\text { Identification of clot- } \\
\text { ting with echo }\end{array}$ & $\begin{array}{c}\text { Clotting in PA } 24 \\
\text { hours later }\end{array}$ & $\begin{array}{c}\text { Hospital stay after } \\
\text { treatment, days }\end{array}$ & $\begin{array}{c}\text { Recurrence of PA at } \\
\text { discharge }\end{array}$ \\
\hline 1 & + & - & 15 & + & + & 6 & - \\
\hline 2 & + & + & 20 & + & + & 4 & - \\
\hline 3 & + & - & 5 & + & + & 5 & - \\
\hline 4 & + & + & 10 & + & + & 2 & - \\
\hline 5 & + & + & 30 & + & + & 12 & - \\
\hline 6 & + & - & 15 & + & + & 3 & - \\
\hline 7 & + & + & 40 & + & + & 4 & - \\
\hline 8 & + & + & 20 & + & + & 9 & - \\
\hline 9 & + & + & 10 & + & + & 8 & - \\
\hline 10 & + & + & 15 & + & + & 3 & - \\
\hline
\end{tabular}

Abbreviations are the same to Table 1.

pseudoaneurysm recurrence or suffered from arterial or venous thrombosis during the pin-point compression.

\subsection{Case 1 (Table 1, No. 4)}

An 83-year-old man underwent percutaneous trans-arterial angioplasty by right femoral artery access through a $6 \mathrm{~F}$ introducer. The introducer was withdrawn 3 hours after the procedure, and astriction was applied for 30 minutes at the puncture site. A pressure bandage was fixed on it for 6 hours. About 24 hours after removal of the fixation, a pulsatile mass with a systolic bruit was found in his right groin. Echographic study revealed a 15 * 20 mm pseudoaneurysm (Figure 3(a)). First, one doctor applied pin-point compression therapy without echoguide. However, the doctor failed to stop the flow through the communication after 50 minutes of compression (Figure 3(b)). During the procedure, the patient did not suffer from pain. Another doctor applied pin-point compression therapy. The doctor initially checked the communication by echography and confirmed the absence of intraluminal flow during finger compression. The patient complained of severe pain during the compression. The pseudoanurysm was repaired with 10 minutes of compression (Figure 3(c)).

\subsection{Case 2 (Table 1, No. 7)}

A 65-year-old man underwent percutaneous coronary angioplasty by right femoral artery access through a 7F introducer. The introducer was withdrawn 2 hours after the procedure, and astriction was applied for 20 minutes at the puncture site. A pressure bandage was fixed on it for 7 hours. About 24 hours after removal of the fixation, the patient presented with pain in the right groin; a pulsatile mass with a systolic bruit was found. Echographic study revealed a $30 * 40 \mathrm{~mm}$ pseudoaneurysm (Figure 4(a)). One doctor applied compression therapy but failed to close the communication after 50 minutes of compression (Figure 4(b)). Another doctor applied pin-point compression therapy while monitoring the flow through the communication. The patient complained of severe pain during the compression. The pseudoanurysm, however, was repaired with 40 minutes of compression (Figure 4(c)).

\section{DISCUSSION}

This study demonstrated that echo-guided pin-point compression can effectively repair an arterial pseudoaneurysm associated with a catheter intervention procedure. The key to this approach is identification of the communication to the artery by color echography and closure of the communication with one-finger compression. Closure of the communication is confirmed by the color Doppler technique. We also observed the process of clot formation in the residual space of the pseudoaneurysm that results in closure of the communication. We were able to successfully repair the pseudoaneurysm using this echo-guided pin-point technique in all of the patients. To our knowledge, this newly developed echoguided pin-point compression is the most effective method for repairing a pseudoaneurysm noninvasively.

The success of this procedure might depend on spontaneously increased activity of the blood coagulation cascade. The extrinsic clotting cascade is initiated at the site of injury in response to the release of tissue factor from the wall of the pseudoaneurysm $[3,4]$. Flow stagnation within the residual lumen may enhance clot formation. Echography showed rapid clot formation in the residual space of the pseudoaneurysm. When we compressed the communication with echo-guide, 7 of the 10 patients experienced severe pain. In our experience, pain can be a sign of successful closure of the communication by pin-point compression. The exact mechanism of pain, however, remains unclear. The key of this procedure is 


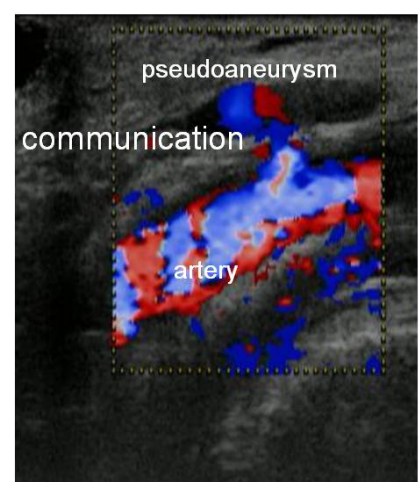

(a)

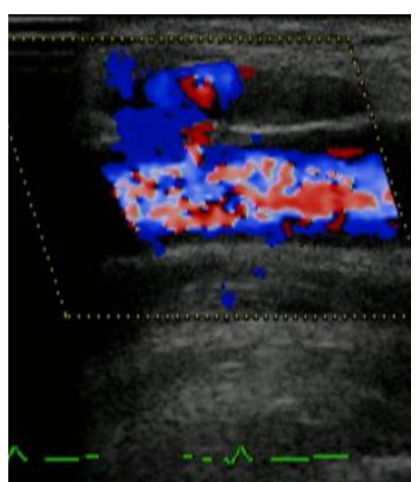

(b)

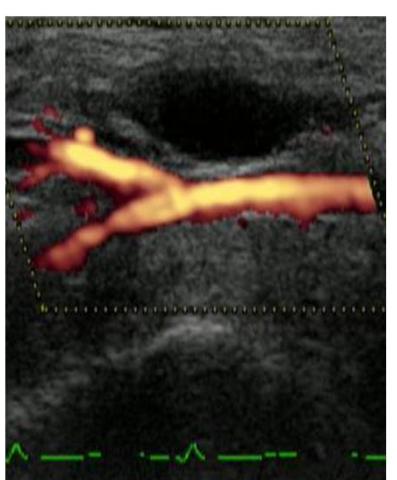

(c)

Figure 3. Echographic findings of case 1 (Table 1, No. 4). (a) Echographic study revealed a pseudoaneurysm (15* $20 \mathrm{~mm}$ in size) after percutaneous transcatheter intervention; (b) One doctor applied the pin-point compression therapy without echo-guide but failed to stop the entry flow after 50 minutes of compression; (c) Another doctor applied echo-guided pin-point compression therapy. Power Doppler echography showed that the pseudoanurysm was repaired by 10 -minute compression.

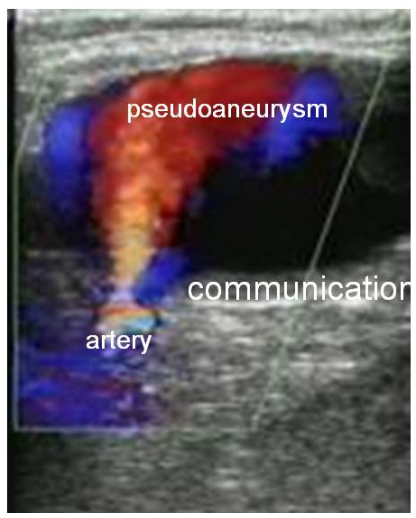

(a)

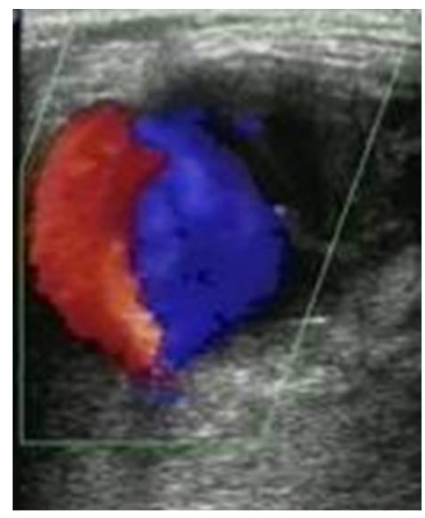

(b)

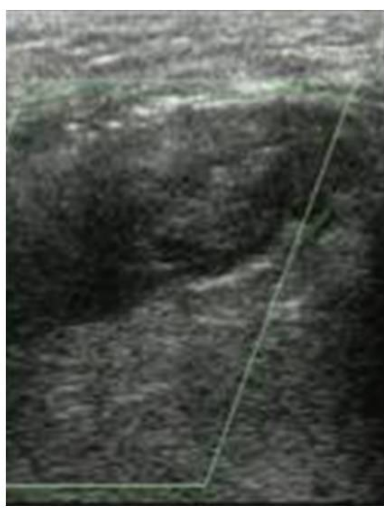

(c)

Figure 4. Echographic findings of case 2 (Table 1, No. 7). (a) Echographic study revealed a pseudoaneurysm (30* $40 \mathrm{~mm}$ in size) after percutaneous coronary intervention; (b) One doctor tried applied compression therapy without echo-guide but failed to stop the entry flow after 50 minutes of compression; (c) Another doctor applied echo-guided pin-point compression therapy. The pseudoanurysm was repaired with 40 -minute compression. Clotting was observed within the pseudoaneurysm.

obstruction of the communication, not associated with compression power. We speculate that compression power is adjustable if echography has shown that the flow in the pseudoaneurysm has disappeared.

The power of compression was reduced when a patient experienced pain, but this was not associated with failure of the procedure. We usually performed no treatment for the pain during pin-point compression because the pain subsides in a few minutes after starting the procedure. For patients who requested the pain control during the pin-point compression, we used mild sedation with hydroxyzine hydrochloride. Two of our patients requested pain control. Light intravenous administration of sedatives was sufficient in both patients.

The echo-guided compression repair technique has been the first line of therapy for a pseudoaneurysm in many institutions, and success rate of this procedure ranged from $75 \%$ to $98 \%$ [2,5-7]. Treatment failures may include cases in which the compression procedure could not successfully close the communication point with a clot because of insufficient blood in the residual lumen to make an obstructive clot. Use of the pin-point compression technique resulted in successful closure of the communication in all of our 10 patients. A randomized study with a larger number of patients is required to confirm the effectiveness of this technique.

Recently, echo-guided injection of thrombin or fibrin into the pseudoaneurysm has been proposed, but its success rate has varied in studies [2,8,9]. The concept of this therapy is similar to our pin-point compression therapy, since both procedures are based on enhancement of clot formation in the lumen to cover the communication of 
the pseudoaneurysm. However, echo-guided injection of thrombin or fibrin has been reported to increase the risk of thrombosis. In contrast, the pin-point compression technique has no risk of such a complication. The pinpoint compression technique might be a safe, valuable, and effective treatment for postcatheter pseudoaneurysms, and it may reduce the need for surgical repair and costs of treatment.

\section{REFERENCES}

[1] McCan, R.L., Schwartz, L.B. and Pieper, K.S. (1991) Vascular complications of cardiac catheterization. Journal of Vascular Surgery, 174, 962-964.

[2] Geoffrey, W. and Wegger, M.D. (2007) Contemporary management of postcatheterization pseudoaneurysms. Circulation, 115, 2666-2674. doi:10.1161/CIRCULATIONAHA.106.681973

[3] Nigel, M., Rachel, E.T. and Nigel, S.K. (2007) Role of the extrinsic pathway of blood coagulation in hemostasis and thrombosis. Arteriosclerosis, Thrombosis, and Vascular Biology, 27, 1687-1693. doi:10.1161/ATVBAHA.107.141911
[4] Weiss, H.J. and Lages, B. (1988) Evidece for tissue factor-dependent activation of the classic extrinsic coagulation mechanism in blood obtained from bleeding time wounds. Blood, 71, 629-635.

[5] Latic, A., Delibegovic, M., Pudic, I., et al. (2011) Noninvasive ultrasound guided compression repair of post puncture femoral pseudoaneurysm. Medical Archives, 65, 113-114.

[6] Eisenberg, L., Paulson, E.K., Kliewer, M.A., et al. (1999) Sonographically guided compression repair of pseudonaeurysms: Further experience from a single institution. American Journal of Roentgenology, 173, 1567-1573.

[7] Steinkamp, H.J., Werk, M. and Felix, R. (2000) Treatment of postinterventional pseudoaneurysms by ultrasound-guided compression. Investive Radiology, 35, 186192. doi:10.1097/00004424-200003000-00005

[8] La Perna, L., Olin, J.W., Goines, D., et al. (2000) Ultrasound-guided thrombin injection for the treatment of postcatheterization pseudoaneurysms. Circulation, 102, 2391-2395. doi:10.1161/01.CIR.102.19.2391

[9] Krueger, K., Zaehringer, M., Strohe, D., et al. (2005) Postcatheterization pseudoaneurysm: Results of US-guided percutaneous thrombin injection in 240 patients. $R a$ diology, 236, 1104-1110. doi:10.1148/radiol.2363040736 